\title{
Self-collimation in $\mathcal{P} \mathcal{T}$-symmetric crystals
}

\author{
W. W. Ahmed, ${ }^{1}$ R. Herrero, ${ }^{1}$ M. Botey, ${ }^{1}$ and K. Staliunas ${ }^{1,2}$ \\ ${ }^{1}$ Departament de Física, Universitat Politècnica de Catalunya (UPC), Colom 11, E-08222 Terrassa, Barcelona, Spain \\ ${ }^{2}$ Institució Catalana de Recerca i Estudis Avançats (ICREA), Passeig Lluís Companys 23, E-08010, Barcelona, Spain
}

(Received 24 November 2016; published 10 May 2017)

\begin{abstract}
We predict the self-collimation phenomena (or equivalently, dynamical localization) in two-dimensional $\mathcal{P} \mathcal{T}$ symmetric complex potentials, where the complex modulation is considered in the transverse, longitudinal, or simultaneously in both directions. Nondiffractive propagation is analytically predicted and further confirmed by numerical integration of a paraxial model. The parameter space is explored to identify the self-collimation regime in crystals with different $\mathcal{P} \mathcal{T}$ symmetries. In addition, we also analyze how the $\mathcal{P} \mathcal{T}$-symmetric potentials determine the energy distribution between spatial modes of the self-collimated beams.
\end{abstract}

DOI: 10.1103/PhysRevA.95.053830

\section{INTRODUCTION}

Diffraction-free propagation of narrow beams, being of fundamental interest in wave dynamics, is especially significant for photonic integrated circuits [1]. Bessel and Airy beams are among the most common examples of nondiffractive beams, which in an ideal case are spatially infinite. In real systems, however, such beams are truncated (since their size and energy are actually finite) and diffraction appears. Therefore, it is essential to control the diffraction of propagating beams through some alternative physical mechanism. Artificial structured materials provide the ability to manipulate the diffraction of propagating beams leading to the self-collimation phenomena. Such self-collimation effect is accounted by the appearance of flat segments in the dispersion curves of propagating modes (the Bloch modes), for certain ranges of frequencies and propagation directions. The quantum mechanical analog of self-collimation is the so-called dynamical localization, which is known to occur in modulated lattices [2-5]. In addition, self-collimation has also been demonstrated in acoustics [6,7], and exciton-polariton condensates [8], though the study of the phenomenon is more advanced in the field of optics. The propagation of nondiffractive beams was initially observed in photonic crystals (PhCs), i.e., structured materials presenting a spatial modulation of the refraction index on the wavelength scale [9-15]. Later, similar beam propagation effects were proposed in gain-loss modulated materials (GLMMs) $[16,17]$. Such materials, instead of periodical modulations of the refractive index, display modulations of gain-loss on the wavelength scale, introducing an anisotropy or angular dependence of the phase velocity and gain, leading to peculiar spatial propagation effects. More recently, self-collimation has also been demonstrated in periodic complex materials [18] and metamaterials [19,20]; besides, flat bands were engineered in thresholdless high-dimensional complex crystals [21] with parity-time $(\mathcal{P} \mathcal{T})$ symmetry.

Indeed, optical periodic structures with $\mathcal{P} \mathcal{T}$ symmetry are attracting an increasing interest to realize new unconventional propagation behaviors [22-24]. These are complex crystals, where real (refractive index) and imaginary (gainloss) modulations of the refractive index are dephased $\pi / 2$, holding potentially technologically important properties such as asymmetrical coupling, asymmetrical reflections, abrupt phase transitions, and loss-induced transparency. In optics, such complex crystals have been already explored in various contexts: field localization [25], unidirectional transmission [26], defect states [27], optical solitons [28], asymmetric chirality [29], and cloaking [30].

In this article, we demonstrate the nondiffractive or self-collimated propagation of light beams in periodic twodimensional (2D) $\mathcal{P} \mathcal{T}$-symmetric crystals. The effect is due to the ability of the complex spatial modulation to tailor the dispersion properties. We perform a comprehensive analysis to assess better conditions for self-collimated beam propagation within such structures. $\mathcal{P} \mathcal{T}$ symmetry is considered here in the transverse, longitudinal, or simultaneously in both directions. The characteristics of the spatial modulation required for the suppression of diffraction are analytically explored in the corresponding parameter space, ranging from the pure $\mathrm{PhC}$ limit to the pure GLMM limit. Numerical simulations based on a paraxial propagation model are performed to confirm the analytical predictions derived from coupled mode equations.

\section{MODEL}

We consider a two-dimensional (2D) spatially periodic lattice described by a complex refractive index function being $\mathcal{P} \mathcal{T}$ symmetric either in the transverse direction $x$, or in the longitudinal direction $z$, or simultaneously in both directions: $\Delta n(x, z)=\Delta n_{\mathrm{Re}}+i \Delta n_{\mathrm{Im}}$, where $\Delta n_{\mathrm{Re}}$ and $\Delta n_{\mathrm{Im}}$ represent the modulation of the refractive index and of the gain-loss, respectively. The modulation is periodic in both directions, transverse and longitudinal, and is on the order of the optical wavelength. The scalar wave propagation equation for an electromagnetic field polarized along $y$, i.e., transversally to the plane and propagation, in the presence of a complex index modulated in the plane $(x, z)$ reads

$$
\frac{n^{2}(x, z)}{c^{2}} \frac{\partial^{2} E}{\partial t^{2}}-\left(\frac{\partial^{2} E}{\partial x^{2}}+\frac{\partial^{2} E}{\partial z^{2}}\right)=0 .
$$

We assume a weak modulation: $n(x, z)=n_{0}+\Delta n$, being $\Delta n(x, z)$ complex, and that the monochromatic field can be described by the slowly varying envelope approximation: $E(x, z, t)=A(x, z, t) e^{-i \omega_{o} t}$ :

$$
\frac{n^{2}(x, z)}{c^{2}}\left(-\omega_{o}^{2} A-2 i \omega_{o} \frac{\partial A}{\partial t}+\frac{\partial^{2} A}{\partial t^{2}}\right)-\left(\frac{\partial^{2}}{\partial x^{2}}+\frac{\partial^{2}}{\partial z^{2}}\right) A=0 .
$$


Under the smallness assumptions $\Delta n \approx O(\varepsilon)$ and $\partial^{2} A /$ $\partial t^{2} \approx O(\varepsilon)$, Eq. (2) at level of $\varepsilon$ may be rewritten as

$$
-2 i \omega_{o} \frac{n^{2}}{c^{2}} \frac{\partial A}{\partial t}=\frac{n^{2}}{c^{2}} \omega_{o}^{2} A+\nabla^{2} A .
$$

Note that Eq. (3) is identical to the Schrödinger equation with a potential, indicating the equivalence between wave dynamics in optical and quantum systems. Next, as we focus on the spatial propagation of beams, we set $\partial A / \partial t=0$ :

$$
\left[1+\frac{2 \Delta n(x, z)}{n_{o}}\right] \frac{\omega_{o}}{2} A+\frac{c^{2}}{2 \omega_{o} n_{o}^{2}} \nabla^{2} A=0 .
$$

We finally consider propagation along $z$, and assume the paraxial approximation on this axis, expressing the slow varying field envelope as $A=A(x, z) e^{i k_{o} z}$ with the propagation equation,

$$
\frac{\partial A}{\partial z}=\frac{i}{2 k_{o}} \frac{\partial^{2} A}{\partial x^{2}}+i k_{o} \frac{\Delta n(x, z)}{n_{o}} A .
$$

Note that the evolution follows along the spatial coordinate $z$, whereas the modulation may occur in $x$ and/or in $z$. In this sense, Eq. (5) is not equivalent to the Schrödinger equation and since the potential can be modulated along the evolution coordinate, the system is not autonomous. However, considering evolution in time, $t$, the system is autonomous [see Eq. (3)].

We shall consider the following three different harmonic modulations and refer to them as $\mathcal{P} \mathcal{T} x, \mathcal{P} \mathcal{T} z$, and $\mathcal{P} \mathcal{T} z x$ :

$$
\begin{aligned}
\Delta n_{\mathcal{P} \mathcal{T} x} & =\left[n_{\mathrm{Re}} \cos \left(q_{\perp} x\right)+i n_{\mathrm{Im}} \sin \left(q_{\perp} x\right)\right] \cos \left(q_{\|} z\right), \\
\Delta n_{\mathcal{P} \mathcal{T} z} & =\left[n_{\mathrm{Re}} \cos \left(q_{\|} z\right)+i n_{\mathrm{Im}} \sin \left(q_{\|} z\right)\right] \cos \left(q_{\perp} x\right), \\
\Delta n_{\mathcal{P} \mathcal{T} z x} & =n_{\mathrm{Re}} \cos \left(q_{\perp} x+q_{\|} z\right)+i n_{\mathrm{Im}} \sin \left(q_{\perp} x+q_{\|} z\right),
\end{aligned}
$$

where the reciprocal lattice vectors of the modulation are $\left( \pm q_{\perp}, \pm q_{\|}\right)$. For convenience, we normalize the transverse coordinate, $x$, to $q_{\perp}^{-1}$ and the longitudinal coordinate, $z$, to $2 k_{0} / q_{\perp}^{2}$-being the wave vector's components $k_{x}, k_{z}$ correspondingly scaled to $q_{\perp}$ and to $q_{\perp}^{2} /\left(2 k_{0}\right)$. Then, the normalized paraxial propagation equation reads

$$
\frac{\partial A}{\partial z}=i \frac{\partial^{2} A}{\partial x^{2}}+i V(x, Q z) A,
$$

where the normalized $\mathcal{P} \mathcal{T}$ potential, $V(x, Q z)$ in Eq. (7), depends on the dimensionless geometrical parameter, $Q=$ $2 k_{0} q_{\|} / q_{\perp}^{2}$, and the normalized real or imaginary amplitudes of the modulation, of Eq. (6), are also scaled to $m_{\mathrm{Re} / \mathrm{m}}=$ $2 n_{\mathrm{Re} / \mathrm{m}} k_{0}^{2} /\left(n_{o} q_{\perp}^{2}\right)$. In order to simplify the study, we assume the condition $m_{\mathrm{Im}}=c-m_{\mathrm{Re}}$, which is orthogonal to the $\mathcal{P} \mathcal{T}$ transition line: $m_{\mathrm{Im}}=m_{\mathrm{Re}}$. In this way, the $\mathcal{P} \mathcal{T}$-symmetric crystal is totally determined by the normalized pair of parameters $\left(m_{\mathrm{Re}}, Q\right)$, within the limits of the paraxial approximation. The index and gain-loss profiles of $\mathcal{P} \mathcal{T} x, \mathcal{P} \mathcal{T} z$, and $\mathcal{P} \mathcal{T} z x$ potentials of Eq. (6) and their complex conjugates, with the corresponding coupling wave vectors, are illustrated in Fig. 1. The coupling behavior depends on the index and gain-loss modulation amplitudes, that can favor the propagation in particular directions with appropriate parameters.
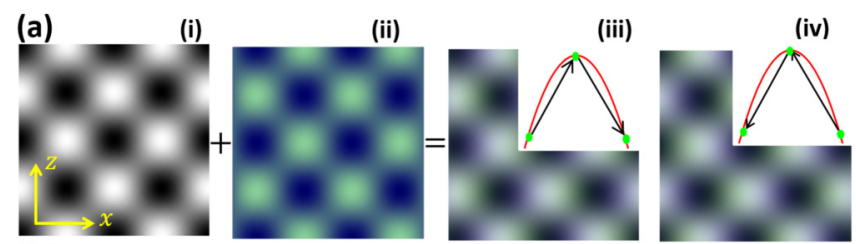

(b)
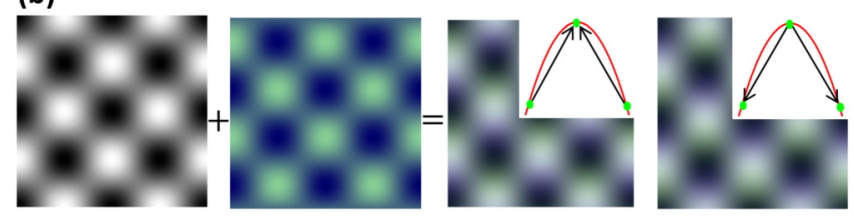

(c)
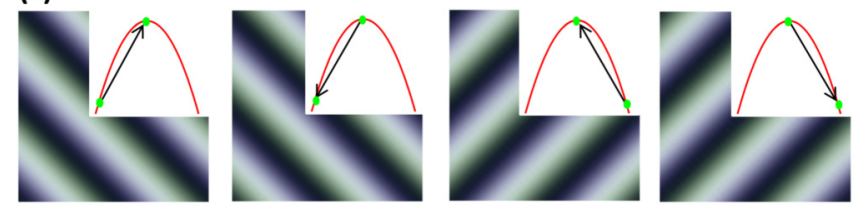

FIG. 1. $\mathcal{P} \mathcal{T}$-symmetric crystals with $\mathcal{P} \mathcal{T}$ modulation in (a) $\mathcal{P} \mathcal{T} x$; (b) $\mathcal{P} \mathcal{T}_{z}$; (c) $\mathcal{P} \mathcal{T}_{z x}$. In (a), (b), refractive index (black is 1 , white is -1 ) and gain-loss [blue (dark gray) is 1, light green (light gray) is -1 ] distributions are shown in (i,ii) while (iii,iv) represent the combination of index and gain-loss profiles into the complex potential and its complex conjugate. In (c), the $\mathcal{P} \mathcal{T} z x$ crystal and its complex conjugate are shown in (i,ii), while (iii,iv) represent the modulation profiles of both $\pi$-rotated $\mathcal{P} \mathcal{T} z x$ crystals. Insets display the corresponding reciprocal lattice vectors for each $\mathcal{P} \mathcal{T}$-symmetric complex crystal to different couplings.

In order to perform an analytical study, we expand the field amplitude in terms of the transverse and longitudinal harmonics of the modulation as

$$
A(x, z)=e^{i k_{x} x} \sum_{l, j} a_{l, j} e^{-i[-(1-j) x+(1+j) \mathrm{j} \mathrm{Q} z]} .
$$

Previous studies on self-collimation in PhCs and GLMMs suggest that such field expansion can be truncated to the three most relevant harmonics $\left(a_{0,0}, a_{0,1}, a_{1,0}\right)$ to understand the complete picture $[15,16]$. Inserting this expansion in the propagation equation, Eq. (7), a system of coupled equations is obtained in the form

$$
\begin{aligned}
& \frac{d a_{0,0}}{d z}=-i k_{x}^{2} a_{0,0}+i m_{1} a_{0,1}+i m_{2} a_{1,0}, \\
& \frac{d a_{0,1}}{d z}=-i\left(k_{x}-1\right)^{2} a_{0,1}+i Q a_{0,1}+i m_{3} a_{0,0}, \\
& \frac{d a_{1,0}}{d z}=-i\left(k_{x}+1\right)^{2} a_{1,0}+i Q a_{1,0}+i m_{4} a_{0,0},
\end{aligned}
$$

where $m_{1}, m_{2}, m_{3}$, and $m_{4}$ are the coupling coefficients calculated from the real and imaginary amplitudes of the considered $\mathcal{P} \mathcal{T}$-symmetric potential. For $\mathcal{P} \mathcal{T} x, m_{1}=m_{4}=$ $\left(m_{\mathrm{Re}}+m_{\mathrm{Im}}\right) / 4$ and $m_{2}=m_{3}=\left(m_{\mathrm{Re}}-m_{\mathrm{Im}}\right) / 4$ while for $\mathcal{P} \mathcal{T} z, m_{1}=m_{2}=\left(m_{\mathrm{Re}}+m_{\mathrm{Im}}\right) / 4$ and $m_{3}=m_{4}=\left(m_{\mathrm{Re}}-\right.$ $\left.m_{\mathrm{Im}}\right) / 4$. Finally, for $\mathcal{P} \mathcal{T} z x$, the $a_{1,0}$ harmonic is decoupled, and the coefficients become $m_{2}=m_{4}=0, m_{3}=\left(m_{\mathrm{Re}}-m_{\mathrm{Im}}\right) / 2$, and $m_{1}=\left(m_{\operatorname{Re}}+m_{\mathrm{Im}}\right) / 2$. Note that since the considered potentials are complex in nature, the coupling between the harmonic components can be both reactive and dissipative, 
(a)

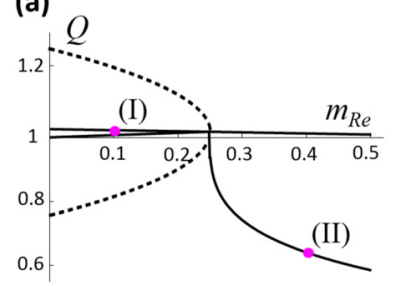

(b)

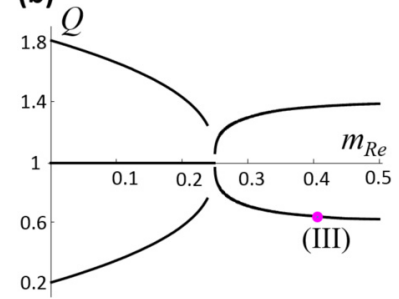

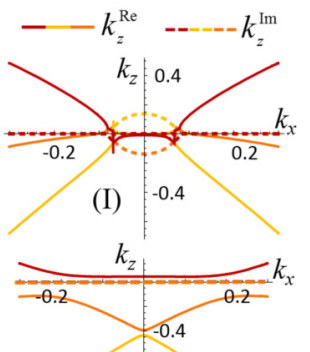

(II) $=-0.8$

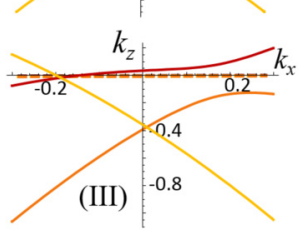

FIG. 2. Three-mode description. (Left) Nondiffraction conditions (solid curves) and nondiffusive propagation (dashed curves) in the $\left(m_{\mathrm{Re}}, Q\right)$ parameter space, being $m_{\mathrm{Im}}=c-m_{\mathrm{Re}}$ for the different potentials where $c=0.5$. (a) $\mathcal{P} \mathcal{T} x$ and $\mathcal{P} \mathcal{T}_{z}$; (b) $\mathcal{P} \mathcal{T} z x$. (Right) Spatial dispersion relations $\left(k_{x}, k_{z}\right)$ for three representative points with nondiffractive propagation corresponding to different parameter sets: (I) $(0.1,0.99)$; (II) $(0.4,0.64)$; (III) $(0.4,0.6)$ the solid (dashed) curves represent the real (imaginary) parts of the wave number, $k_{z}^{\mathrm{Re}}, k_{z}^{\mathrm{Im}}$.

determining the propagation properties within such structures. However, $\mathcal{P} \mathcal{T}$-symmetric structures may be reduced to two limiting cases corresponding to PhC-like and a GLMM-like media, at each side of the $\mathcal{P} \mathcal{T}$-transition condition $\left(m_{\mathrm{Re}}=\right.$ $m_{\text {Im }}=c / 2$ ). The PhC-like side of the $\mathcal{P} \mathcal{T}$-symmetry transition corresponds to $m_{\mathrm{Re}}>m_{\mathrm{Im}}$, while for $m_{\mathrm{Re}}<m_{\mathrm{Im}}$ the system behavior is GLMM-like. For PhC-like media, the reactive coupling pushes the eigenvalues apart, near the resonances between coupled modes, opening band gaps in the spatial dispersion. The spatial evolution of a beam propagating in the $z$ direction is determined by the on-axis modes $\left(k_{x} \approx 0\right)$ in which dispersion is modified near the resonance of three harmonics, given by $Q \approx 1$, as follows from Ref. [15]. Moreover, all eigenvalues are real valued and provide the propagation wave number of the Bloch mode [see the dispersion relations in Figs. 2(b) (II) and 2(b) (III)]. On the other hand, for GLMMlike media, eigenvalues are complex conjugated; thus their real parts lock while a nonzero imaginary part develops [see Fig. 2(b) (I)]. The curvature of the real and imaginary spatial dispersion $\left(k_{z}^{\mathrm{Re} / \mathrm{Im}}, k_{x}\right)$ determines the diffraction and diffusion of light, respectively. Therefore, nondiffractive or nondiffusive regimes can be respectively obtained imposing a zero value to the second derivative either of the real or the imaginary part of spatial dispersion. Nondiffractive propagation, also called self-collimation, can be achieved for specific parameter sets for both PhC-like and GLMM-like media, although, in the last case, diffusion still persists which also spreads the propagating beam.

First, we consider the potential being $\mathcal{P} \mathcal{T}$ symmetric in only one direction, either in the transverse or the longitudinal direction, while the modulation in the other direction has a real amplitude $(\mathcal{P} \mathcal{T} x$ or $\mathcal{P} \mathcal{T} z)$. The coupling symmetry among the harmonics ensures the same eigenvalues for both cases,

although eigenvectors are asymmetric for $\mathcal{P} \mathcal{T} x$ potentials and symmetric for $\mathcal{P} \mathcal{T} z$ potentials. The results for nondiffractive and nondiffusive beam propagation in the parameter space $\left(m_{\mathrm{Re}}, Q\right)$, are provided in Fig. 2(a). Note the lack of crossing points of the nondiffractive and nondiffusive curves meaning the nonpossible beam self-collimation in GLMM-like media. Therefore, self-collimation results for the $\mathcal{P} \mathcal{T} x$ or $\mathcal{P} \mathcal{T} z$ potential only appear in the $\mathrm{PhC}$-like regime, where the system has no intrinsic diffusion. The corresponding real-valued dispersion, exhibiting a flat nondiffractive mode, is shown in Fig. 2 (II) [the red (dark gray) curve]. We also analyze the case where the potential is $\mathcal{P} \mathcal{T}$ symmetric in both transverse and longitudinal direction $(\mathcal{P} \mathcal{T} z x)$ and determine the nondiffractive regime in parameter space $\left(m_{\mathrm{Re}}, Q\right)$, as depicted in Fig. 2(b). Note that in this case, the red curve corresponding to point III is a nondiffractive mode $\left(\partial^{2} k_{z} / \partial k_{x}^{2}=0\right)$; however, it has nonzero refraction $\left(\partial k_{z} / \partial k_{x} \neq 0\right)$.

\section{NUMERICAL ANALYSIS}

We have analytically predicted that $\mathcal{P} \mathcal{T}$-symmetric modulations, of gain-loss and refractive index, allow tailoring of the dispersion properties and lead to nondiffractive and nondiffusive beam propagation for particular modulation parameters, as shown in Fig. 2. In order to verify the analytical results, we numerically propagate a Gaussian beam, $A(x)=$ $\exp \left(-x^{2} / w_{0}^{2}\right)$, through these complex structures and determine the evolution of the field intensity in either PhC-like or GLMM-like situations. After a sufficient propagation length,
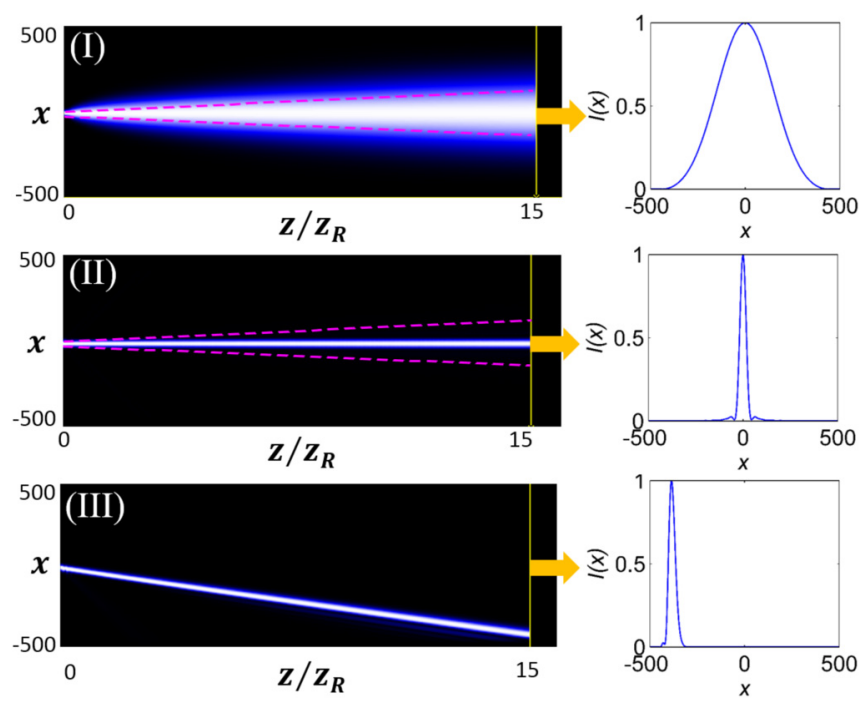

FIG. 3. Propagation of a Gaussian beam through $\mathcal{P} \mathcal{T}$-symmetric media for a normalized propagated length of $z=15 z_{R}, z_{R}$ being the Rayleigh length. (Left) Field intensity evolution for the three particular parameter sets of Fig. 2, exhibiting (I) nondiffractive but diffusive propagation for a $\mathcal{P} \mathcal{T} x$ GLMM-like case, (II) nondiffractive propagation for a $\mathcal{P} \mathcal{T} x$ PhC-like case, and (III) nondiffractive propagation for a $\mathcal{P} \mathcal{T} z x$ PhC-like case. (Right) Corresponding field intensity transversal profiles at the propagation length $z=15 z_{R}$. The peak amplitude in each transverse section is normalized to unity. The initial Gaussian beam width is $w_{0}=20$ and $z_{R} \approx 100$. The dotted line in (I,II) represents the free-space diffracted beam, shown for comparison. 
the beam width is calculated using a knife-edge method, by filtering out the higher spatial frequencies, revealing a perfect agreement with the analytical predictions. The evolution of the field intensity, for the representative points (I, II, III) of Fig. 2, is shown in Fig. 3. An initial Gaussian beam experiences a nondiffractive but diffusive beam propagation through the GLMM-like potential with parameter set (I), while nondiffractive beam propagation is achieved for parameter sets (II) and (III) corresponding to PhC-like cases. Transverse modes with wave number $k_{x}>0.5$ are filtered out to remove the transverse modulation of the potential for a clearer representation of the intensity profiles (see the right column of Fig. 3). The evolution of the filtered beam profile is the same for the $\mathcal{P} \mathcal{T} x$ and the $\mathcal{P} \mathcal{T} z$ cases, as expected from the coincidence of eigenvectors of the analytical curves. The cases (I) and (II) in Fig. 3 have been calculated using the $\mathcal{P} \mathcal{T} x$ potential.

Next, we numerically explore the parameter space, $\left(m_{\mathrm{Re}}, Q\right)$, to identify the self-collimation regime for $\mathcal{P} \mathcal{T} x$ (or $\mathcal{P} \mathcal{T}_{z}$ ) and $\mathcal{P} \mathcal{T} z x$ potentials. The results are summarized in Fig. 4, which depicts the map of the beam width normalized to the initial width $\left(w / w_{0}\right)$. For the $\mathcal{P} \mathcal{T} x$ potential, the parameter space is explored for both limits of the PhC-like and the GLMM-like media. We note that the $\mathcal{P} \mathcal{T} z$ potential leads to exactly the same width distributions in the parameter space as the $\mathcal{P} \mathcal{T} x$ case; however, it shows different beam dynamics, due to the symmetrical mode coupling and amplitudes of transverse modes. The white islands in the Fig. 4(a) map correspond to the self-collimation regime, for which the initial beam
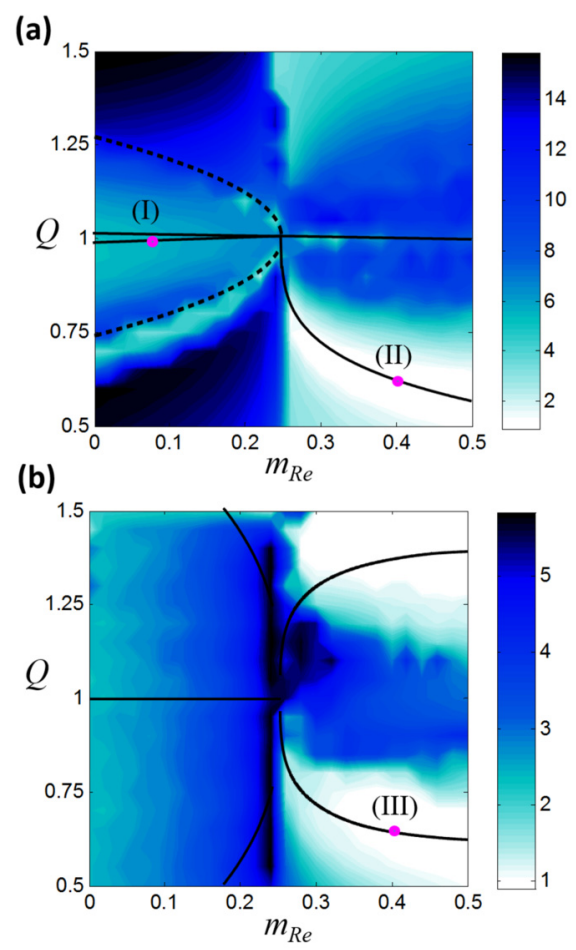

FIG. 4. Beam width maps in parameter space, $\left(m_{\mathrm{Re}}, Q\right)$. Numerical beam width $\left(w / w_{0}\right)$ after a long propagation $\left(z=5 z_{R}\right)$ of a Gaussian beam for different $\mathcal{P} \mathcal{T}$-symmetric potentials: (a) $\mathcal{P} \mathcal{T} x$; (b) $\mathcal{P} \mathcal{T} z x$. The white islands represent the self-collimation regimes. The solid and dotted curves show the analytical nondiffractive and nondiffusive conditions provided for comparison. width is kept for a long propagation length. For the $\mathcal{P} \mathcal{T} z x$ potential, two distinct self-collimation regions are found, both in the PhC-like limit, $m_{\mathrm{Re}} \geqslant c / 2$ [see Fig. 4(b)]. The two white islands in Fig. 4(b) correspond to the nondiffractive but refractive propagating mode, analytically predicted in Fig. 2(b); however, modes lying in the bottom island carry more energy than the ones with higher $Q$. In the GLMM-like case, the incident beam always broadens due to the presence of diffusion even in the self-collimation condition. Moreover, the field is enhanced in other directions by modes with positive effective gain and always positive diffraction. The analytically predicted curves for nondiffractive and nondiffusive conditions are also superimposed onto the numerically calculated maps for comparison. The numerical results evidence a perfect agreement with the analytical predictions, providing confirmation of the self-collimation regimes.

\section{MODE DYNAMICS}

Furthermore, we analyze the dynamics of beam propagation in the self-collimation regime, for $\mathcal{P} \mathcal{T} x$ and $\mathcal{P} \mathcal{T} z$ potentials. As previously indicated, both systems hold the same eigenvalues with, however, different eigenvectors, which lead to the different mode coupling. We start by analytically determining the eigenvector components of the $\mathcal{P} \mathcal{T} x$ potential, for a given parameter set $\left(m_{\mathrm{Re}}, Q\right)$, lying on the lower nondiffractive eigenvalue branch of Fig. 2(a), and normalized to the fundamental harmonic as $\left|a_{+}(k)\right|=\left|a_{1,0}(k) / a_{0,0}(k)\right|$ and $\left|a_{-}(k)\right|=\left|a_{0,1}(k) / a_{0,0}(k)\right|$. Such components are depicted in Fig. 5(a) (i); the same values for a corresponding complex conjugated $\mathcal{P} \mathcal{T} x$ potential are also provided in Fig. 5(b) (i). It is obvious from the spectra that the mode coupling is asymmetric (a)
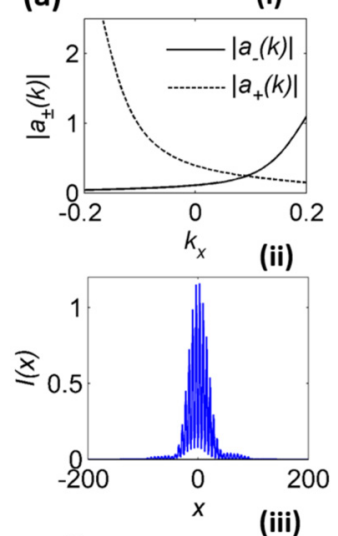

(iii)

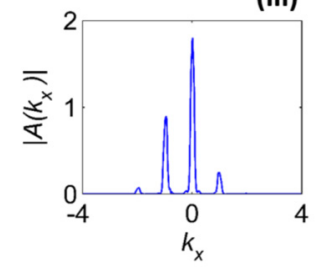

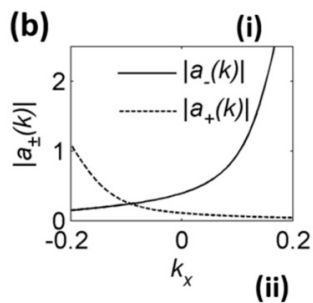

(ii)
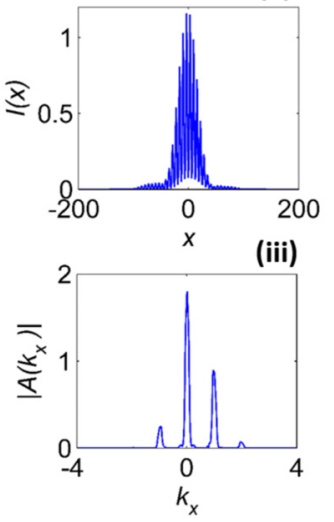

FIG. 5. Dynamics analysis of a $\mathcal{P} \mathcal{T} x$ potential (a) and its complex conjugate (b). (i) Normalized eigenvector components of nondiffracted branches of eigenvalues; (ii) field intensity profiles of the propagated Gaussian beam; (iii) corresponding spatial field spectra. Calculations correspond to the parameter set $(0.32,0.71)$ after propagation length $z \approx 6 z_{R}$. 
in both $\mathcal{P} \mathcal{T} x$ potentials, and that the dominant side-band mode is interchanged in complex conjugated potentials.

Next, we compare such results with the numerically calculated spatial spectra, $\left|A\left(k_{x}\right)\right|$ of Gaussian beams propagated in such potentials [see the nonfiltered beam profiles in Figs. 5(a) (ii) and 5(b) (ii)]. The numerically calculated spectra, and also the corresponding beam profiles, exhibit the predicted asymmetry, being $\left|A\left(k_{x}=-1\right)\right|>\left|A\left(k_{x}=1\right)\right|$ as illustrated in Fig. 5(a) (iii) for a given nondiffractive parameter set [and $\left|A\left(k_{x}=-1\right)\right|<\left|A\left(k_{x}=1\right)\right|$ for the complex conjugated $\mathcal{P} \mathcal{T} x$ potential; see Fig. 5(b) (iii)]. Therefore, in complex conjugated potentials, the energy flows in opposite directions due to the unidirectional coupling, as schematically represented in Figs. 1(a) (iii) and 1(a) (iv).

On the other hand, for $\mathcal{P} \mathcal{T} z$ systems we predict a symmetric mode coupling, $\left|a_{+}(k)\right|=\left|a_{-}(k)\right|$, such components being larger for the complex conjugated potential [see Figs. 6(a) (i) and 6(a) (ii)]. In this case, the symmetric spatial spectra denote a symmetric energy distribution on the side bands-being either reduced or enhanced, depending on the coupling sense [see Figs. 1(b) (iii) and 1(b) (iv)]. The comparison with the numerically obtained spatial spectra also shows a good agreement in this case; see Figs. 6(a) (ii) and 6(a) (iii), and 6(b) (ii) and 6(b) (iii). The spectral analysis clearly indicates that the energy distribution between modes can be manipulated by $\mathcal{P} \mathcal{T}$-symmetric potentials, either asymmetrically for the $\mathcal{P} \mathcal{T} x$ or symmetrically for $\mathcal{P} \mathcal{T} z$ potentials. The interplay of the two transverse modulations, with appropriate parameters, favors the propagation in a particular direction.

The dynamics of the spatial modes amplitude of selfcollimated beams is now investigated, for both $\mathcal{P} \mathcal{T} x$ and (a)

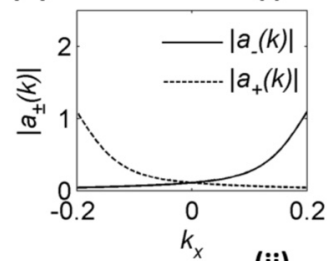

(ii)
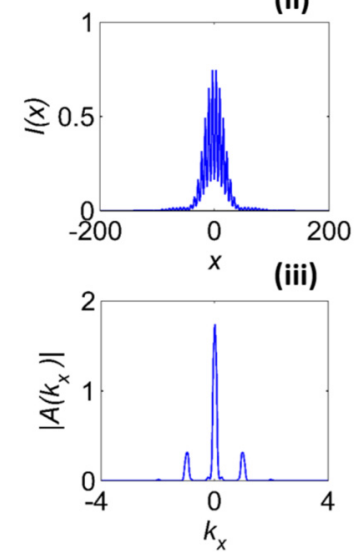

(b)

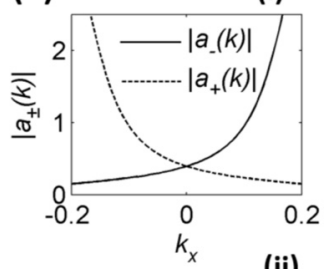

(ii)
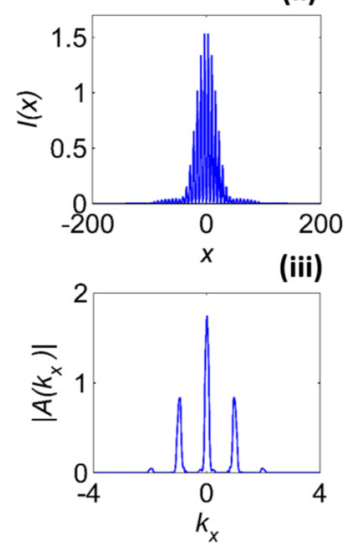

FIG. 6. Dynamics analysis of a $\mathcal{P} \mathcal{T} z$ potential (a) and its complex conjugate (b). (i) Normalized eigenvector components of nondiffracted branches of eigenvalues; (ii) field intensity profiles of the propagated Gaussian beam; (iii) corresponding spatial field spectra. Calculations correspond to parameter set $(0.32,0.71)$ after propagation length $z \approx 6 z_{R}$.
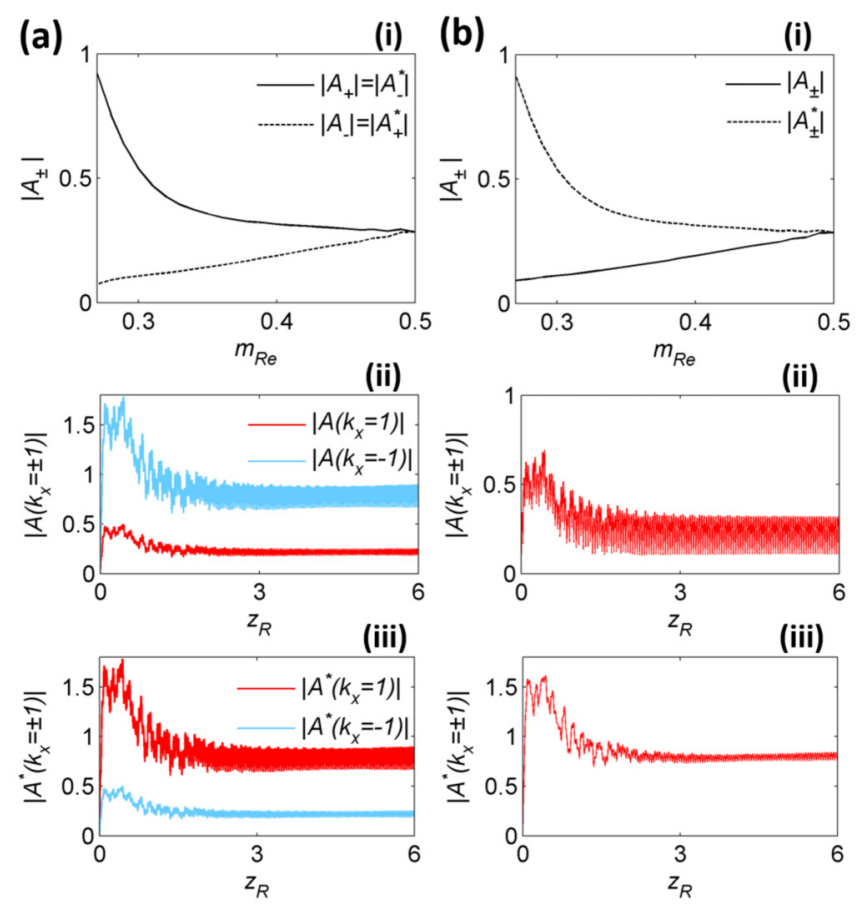

FIG. 7. Numerical study of the dynamics of the spectral field components for a $\mathcal{P} \mathcal{T} x$ (a) and $\mathcal{P} \mathcal{T}$ z (b) potentials. (i) Normalized spectral field amplitudes $\left|A_{ \pm}\right|$, along the nondiffractive curves of Fig. 2, for the potentials and their corresponding complex conjugated. Evolution of the spectral field components $\left|A\left(k_{x}= \pm 1\right)\right|$ in propagation for the considered complex potentials (ii) and their complex conjugates (iii), for the parameter set $(0.32,0.71)$.

$\mathcal{P} \mathcal{T} z$ potentials. We determine the mean values of the spectral field components of the numerically propagated field, along the nondiffractive curves of Fig. 2(a), and normalize the side-band amplitudes to the central mode amplitude as $\left|A_{ \pm}\right|=$ $\left\langle\left|A\left(k_{x}= \pm 1\right)\right|\right\rangle /\left\langle\left|A\left(k_{x}=0\right)\right|\right\rangle$ (see Fig. 7). For the $\mathcal{P} \mathcal{T} x$ case, the mode coupling is asymmetric, and indeed $\left|A_{+}\right|$and $\left|A_{-}\right|$show different values, as depicted in Fig. 7(a) (i) for the same nondiffractive parameter set of Fig. 5. However, mode amplitudes, after undergoing some transition, exhibit periodic oscillations, as illustrated in Fig. 7(a) (ii), attributed to the longitudinal periodicity of the potential. Note that for the complex conjugated $\mathcal{P} \mathcal{T} x$ potential, the spectral field amplitudes are only reversed [see Fig. 7(a) (iii)]. On the other hand, for the $\mathcal{P} \mathcal{T} z$ case the symmetric coupling leads to equal spectral amplitudes of the spatial modes, $\left|A_{+}\right|=\left|A_{-}\right|$. This also holds for the corresponding complex conjugated potential, for which the side-band energy is enhanced, as illustrated in Figs. 7(b) (ii) and 7(b) (iii).

\section{v. CONCLUSIONS}

We propose nondiffractive propagation of narrow beams in $\mathcal{P} \mathcal{T}$-symmetric crystals where gain-loss and refractive index modulations are phase shifted by a quarter of the spatial period. We analyze $2 \mathrm{D}$ modulated systems, holding $\mathcal{P} \mathcal{T}$ symmetry either in the transverse $(\mathcal{P} \mathcal{T} x)$, longitudinal $(\mathcal{P} \mathcal{T} z)$, or simultaneously in both directions $(\mathcal{P} \mathcal{T} z x)$. Such complex modulations may influence the system dispersion leading to 
a complete suppression of diffraction and diffusion. We show that such complex crystals offer the possibility to suppress diffraction in the limiting cases of PhC-like and GLMMlike behaving crystals. However, nondiffractive beams in GLMM-like systems always experience self-broadening due to intrinsic diffusion. We explore the parameter space to determine the self-collimation regimes for $\mathcal{P} \mathcal{T} x, \mathcal{P} \mathcal{T} z$, and $\mathcal{P} \mathcal{T} z x$ potentials. The analytical conditions for nondiffractive and nondiffusive beam propagation are in perfect agreement with numerical simulations performed under a paraxial approximation. Finally, we analyze the beam dynamics in the self-collimation regime. The symmetric and asymmetric coupling in self-collimated beams opens the way to control the energy distribution among spatial modes by $\mathcal{P} \mathcal{T}$-symmetric potentials. The predicted self-collimation effect may be useful to shape the beam profile in $\mathcal{P} \mathcal{T}$-symmetric integrated optics.

\section{ACKNOWLEDGMENTS}

We acknowledge financial support by Spanish Ministerio de Economía, Industria y Competitividad through Project No. FIS2015-65998-C2-1-P, NATO Project No. SPS-985048, and Erasmus Mundus Doctorate Program Europhotonics (Grant No. 159224-1-2009-1-FR-ERA MUNDUS-EMJD).
[1] D. W. Prather, S. Shi, J. Murakowski, G. J. Schneider, A. Sharkawy, C. Chen, B. Miao, and R. Martin, J. Phys. D 40, 2635 (2007).

[2] S. Longhi, M. Marangoni, M. Lobino, R. Ramponi, P. Laporta, E. Cianci, and V. Foglietti, Phys. Rev. Lett. 96, 243901 (2006).

[3] H. Lignier, C. Sias, D. Ciampini, Y. Singh, A. Zenesini, O. Morsch, and E. Arimondo, Phys. Rev. Lett. 99, 220403 (2007).

[4] I. L. Garanovich, S. Longhi, A. A. Sukhorukov, and Y. S. Kivshar, Phys. Rep. 518, 1 (2012).

[5] K. Staliunas, R. Herrero, and G. J. de Valcarcel, Phys. Rev. E 73, 065603(R) (2006).

[6] V. Espinosa, V. J. Sanchez-Morcillo, K. Staliunas, I. PerezArjona, and J. Redondo, Phys. Rev. B 76, 140302(R) (2007).

[7] I. Pérez-Arjona, V. J. Sánchez-Morcillo, J. Redondo, V. Espinosa, and K. Staliunas, Phys. Rev. B 75, 014304 (2007).

[8] H. Deng, H. Haug, and Y. Yamamoto. Rev. Mod. Phys. 82, 1489 (2010).

[9] R. Zengerle, J. Mod. Opt. 34, 1589 (1987).

[10] H. Kosaka, T. Kawashima, A. Tomita, M. Notomi, T. Tamamura, T. Sato, and S. Kawakami, Appl. Phys. Lett. 74, 1212 (1999).

[11] J. Witzens, M. Lonacar, and A. Scherer, IEEE J. Sel. Top. Quantum Electron. 8, 1246 (2002).

[12] D. Chigrin, S. Enoch, C. Sotomayor Torres, and G. Tayeb, Opt. Express 11, 1203 (2003).

[13] R. Iliew, C. Etrich, U. Peschel, F. Lederer, M. Augustin, H.-J. Fuchs, D. Schelle, E.-B. Kley, S. Nolte, and A. Tunnermann, Appl. Phys. Lett. 85, 5854 (2004).

[14] D. W. Prather, S. Shi, D. M. Pustai, C.A. Chen, S. Venkataraman, A. Sharkawy, G. J. Schneider, and J. Murakowski, Opt. Lett. 29, 50 (2004).

[15] K. Staliunas and R. Herrero, Phys. Rev. E 73, 016601 (2006).
[16] K. Staliunas, R. Herrero, and R. Vilaseca, Phys. Rev. A 80, 013821 (2009).

[17] M. Botey, R. Herrero, and K. Staliunas, Phys. Rev. A 82, 013828 (2010).

[18] R. Herrero, M. Botey, and K. Staliunas, Phys. Rev. A 89, 063811 (2014).

[19] R. Polles, E. Centeno, J. Arlandis, and A. Moreau. Opt. Express 19, 6149 (2011).

[20] G. D’Aguanno, N. Mattiucci, and M. J. Bloemer, J. Opt. Soc. Am. B 25, 236 (2008).

[21] A. Cerjan, A. Raman, and S. Fan, Phys. Rev. Lett. 116, 203902 (2016).

[22] A. Guo, G. J. Salamo, D. Duchesne, R. Morandotti, M. VolatierRavat, V. Aimez, G. A. Siviloglou, and D. N. Christodoulides, Phys. Rev. Lett. 103, 093902 (2009).

[23] C. E. Ruter, K. G. Makris, R. El-Ganainy, D. N. Christodoulides, M. Segev, and D. Kip, Nat. Phys. 6, 192 (2010).

[24] A. Regensburger, C. Bersch, M. A. Miri, G. Onishchukov, D. N. Christodoulides, and U. Peschel, Nature 488, 167 (2012).

[25] C. Hang, Y. V. Kartashov, G. Huang, and V. V. Konotop, Opt. Lett. 40, 2758 (2015).

[26] Z. Lin, H. Ramezani, T. Eichelkraut, T. Kottos, H. Cao, and D. N. Christodoulides, Phys. Rev. Lett. 106, 213901 (2011).

[27] A. Regensburger, M.-A. Miri, C. Bersch, J. Nager, G. Onishchukov, D. N. Christodoulides, and U. Peschel, Phys. Rev. Lett. 110, 223902 (2013).

[28] M. Wimmer, A. Regensburger, M.-A. Miri, C. Bersch, D. N. Christodoulides, and U. Peschel, Nature Commun. 6, 7782 (2015).

[29] M. Turduev, M. Botey, I. Giden, R. Herrero, H. Kurt, E. Obzay, and K. Staliunas, Phys. Rev. A 91, 023825 (2015).

[30] X. Zhu, L. Feng, P. Zhang, X. Yin, and X. Zhang, Opt. Lett. 38, 2821 (2013). 